\title{
Gravitating multidefects from higher dimensions
}

\author{
Massimo Giovannini* \\ Centro "Enrico Fermi", Compendio del Viminale, Via Panisperna 89/A, 00184 Rome, Italy \\ and Department of Physics, Theory Division, CERN, 1211 Geneva 23, Switzerland
}

(Received 12 December 2006; published 20 March 2007)

\begin{abstract}
Warped configurations admitting pairs of gravitating defects are analyzed. After devising a general method for the construction of multidefects, specific examples are presented in the case of higherdimensional Einstein-Hilbert gravity. The obtained profiles describe diverse physical situations such as (topological) kink-antikink systems, pairs of nontopological solitons, and bound configurations of a kink and of a nontopological soliton. In all the mentioned cases the geometry is always well behaved (all relevant curvature invariants are regular) and tends to five-dimensional anti-de Sitter space-time for large asymptotic values of the bulk coordinate. Particular classes of solutions can be generalized to the framework where the gravity part of the action includes, as a correction, the Euler-Gauss-Bonnet combination. After scrutinizing the structure of the zero modes, the obtained results are compared with conventional gravitating configurations containing a single topological defect.
\end{abstract}

DOI: 10.1103/PhysRevD.75.064023

PACS numbers: 04.50.+h, 05.45.Yv, 11.10.Kk, 11.25.Mj

\section{FORMULATION OF THE PROBLEM}

Solitons represent an important class of phenomena both in classical and quantum field theory. Solitonic solutions include monopoles, skyrmions, vortices, and kinks [1]. It is well known that in $(1+1)$-dimensional field theories (static) topological solitons can arise in combination with an appropriately nonlinear interaction potential [2,3]. These solutions are customarily called kinks and they may arise either in the case of polynomial potentials or in the case of sine-Gordon potentials [1,4,5]. In $(1+1)$ dimensions, spatial infinity consists of two points (i.e. $\pm \infty$ ) and the kink solution typically interpolates between two minima of the underlying potential. The topological charge of the kink is positive. If the kink solution exists, there will also typically exist the related antikink solution whose topological charge will be opposite to the one of the kink. In recent years general methods have been devised for the analysis of the complicated nonlinear problem arising in the case of $(1+1)$-dimensional multidefect systems [6-10].

It is also well known that, in $(1+1)$ dimensions, nontopological solitons can occur. These objects have vanishing topological charge since the field profile vanishes for large absolute value of the spatial coordinate [11].

It is finally known that, with the appropriate field content, multidefects do arise in $(1+1)$ dimensions $[2,3,8,10]$. These solutions include, for instance, bound states of a kink and of an antikink and the so-called trapping bag solutions $[3,12]$ where a nontopological profile is supplemented by a kink (or an antikink) profile.

There are mathematical and physical analogies between the defects of $(1+1)$-dimensional field theories and the gravitating solitonic solutions of five-dimensional gravity. In the latter case the rôle of the spatial coordinate is played

*Electronic address: massimo.giovannini@cern.ch by the fifth dimension parametrizing the field profile in the bulk space-time. Along this perspective it is known that gravitating kink solutions may arise, for instance, in the context of five-dimensional Einstein-Hilbert gravity. Different groups analyzed single kink solutions in higherdimensional geometries. In particular examples are known both in flat five-dimensional space-time [13] (see also [14]), and in five-dimensional (warped) space-times [1520] (see also [21,22] for an interesting perspective). While the compatibility of these configurations with higherdimensional Einstein-Hilbert gravity has been ascertained, interesting generalizations contemplate the coupling of the scalar degree of freedom to the curvature [23-25], the inclusion of Gauss-Bonnet self-interactions [26-30], the addition of more than one internal (warped) dimension (see, for instance, [31-33]). While the features of the geometry may be diverse, it is certainly plausible (even if not mandatory) that gravitating kink solutions lead to fivedimensional anti-de Sitter geometry $\left(\mathrm{AdS}_{5}\right.$ in the following) for large absolute values of the bulk coordinate. In a related framework it is also possible to obtain kink profiles whose related geometry leads, for large absolute values of the bulk radius, to five-dimensional Minkowski spacetime. Moreover, single defects with nontopological features have been studied in [34]. It should be stressed that gravity is an essential ingredient for all the fivedimensional solitonic solutions mentioned in the present paragraph. By contrast, in $(1+1)$ dimensions, gravitational interactions are absent. This simple observation is the root of the physical differences occurring in the structure of the zero modes of the system.

Indeed, gravitating solutions containing a single defect are rather intriguing since they may be used to localize fluctuations of various spin in five-dimensional gravity. They constitute a viable example of (static) brane models where the thickness of the brane does not vanish as it happens, instead in the Randall-Sundrum setup [35,36]. 
The problem of localization of the fluctuations of singledefect models has been addressed in a number of ways. In $[19,20,37]$ a fully gauge-invariant formalism has been proposed and, subsequently, useful gauge-dependent approaches also have been developed [38,39] (see also [40]).

The problem to be discussed in the present paper can be formulated in analogy with the case of $(1+$ 1)-dimensional field theories. While we do know that multidefects are present in $(1+1)$ dimensions, it would be interesting to know if multidefects may arise in warped geometries. Consider, therefore, the simplest fivedimensional framework allowing for gravitating multidefects and described by the action ${ }^{1}$

$$
\begin{aligned}
S_{\mathrm{EH}}= & \int d^{5} x \sqrt{|G|}\left[-\frac{R}{2 \kappa}+\frac{1}{2} G^{A B} \partial_{A} \varphi \partial_{B} \varphi\right. \\
& \left.+\frac{1}{2} G^{A B} \partial_{A} \chi \partial_{B} \chi-W(\varphi, \chi)\right],
\end{aligned}
$$

where $\kappa=8 \pi / M_{5}^{3}, G_{A B}$ is the five-dimensional metric tensor, and $R$ the five-dimensional Ricci scalar. In Eq. (1.1) $\phi$ and $\chi$ are two scalar degrees of freedom and $W(\phi, \chi)$ is the potential containing both the selfinteractions of $\phi$ and $\chi$ as well as their mutual interactions. It will be shown that solutions containing pairs of gravitating defects are a common feature of the theory defined by Eq. (1.1) and can be obtained with an appropriate (but rather general) constructive procedure. The discussion presented here is limited to a pair of defects; however, it seems rather plausible to extend the discussion to an even larger number of fields.

It was recently pointed out that kink-antikink solutions as well as trapping bag solutions may arise in the presence of suitably tuned Gauss-Bonnet corrections to the EinsteinHilbert action [41]. In the present analysis those considerations will be extended and it will be shown, in particular, that Gauss-Bonnet corrections are a useful addition but they are not crucial for the existence of gravitating multidefects. After discussing the physical properties of the new solutions containing pairs of defects it will be interesting also to develop the gauge-invariant approach proposed in $[19,20,37]$ to include the case of configurations containing more than one defect.

The plan of the present paper is then the following. In Sec. II the framework of the present analysis will be explained. In Sec. III, after developing a rather general method for the integration of the system, kink-antikink configurations will be specifically analyzed. Section IV deals primarily with bound systems of topological and nontopological solitons. The analysis of the zero modes of the system is contained in Sec. V. Section VI contains

\footnotetext{
${ }^{1}$ Latin (uppercase) letters run over the higher-dimensional space-time while Greek (lowercase) indices are defined over the four-dimensional space-time.
}

the concluding remarks and a summary of the main findings of the present investigation.

\section{THE GENERAL FRAMEWORK}

Consider first the case of five-dimensional EinsteinHilbert gravity characterized by the action (1.1). The field equations derived from Eq. (1.1) simply become

$$
\begin{aligned}
& R_{A B}-\frac{1}{2} G_{A B} R=\kappa T_{A B}, \\
& G^{A B} \nabla_{A} \nabla_{B} \phi+\frac{\partial W}{\partial \phi}=0, \\
& G^{A B} \nabla_{A} \nabla_{B} \chi+\frac{\partial W}{\partial \chi}=0,
\end{aligned}
$$

where $R_{A B}$ is the Ricci tensor and $\nabla_{A}$ is the covariant derivative constructed from the five-dimensional metric $G_{A B}$. The energy-momentum tensor of the system, $T_{A B}$, is given, according to Eq. (1.1) by

$$
\begin{aligned}
T_{A B}= & \partial_{A} \phi \partial_{B} \phi+\partial_{A} \chi \partial_{B} \chi-G_{A B}\left[\frac{G^{M N}}{2} \partial_{M} \phi \partial_{N} \phi\right. \\
& \left.+\frac{G^{M N}}{2} \partial_{M} \chi \partial_{N} \chi-W(\phi, \chi)\right] .
\end{aligned}
$$

Consider now, in particular, the five-dimensional (warped) line element

$$
d s^{2}=a^{2}(w)\left[\eta_{\mu \nu} d x^{\mu} d x^{\nu}-d w^{2}\right],
$$

where $w$ is the bulk coordinate, $a(w)$ the warp factor, and $\eta_{\mu \nu}$ the four-dimensional Minkowski metric. In the case described by Eq. (2.5), Eqs. (2.1), (2.2), and (2.3) become, respectively,

$$
\begin{gathered}
\mathcal{H}^{\prime}+\mathcal{H}^{2}=-\frac{\kappa}{3}\left[\frac{\phi^{\prime 2}}{2}+\frac{\chi^{\prime 2}}{2}+W a^{2}\right], \\
\mathcal{H}^{2}=\frac{\kappa}{6}\left[\frac{\phi^{\prime 2}}{2}+\frac{\chi^{\prime 2}}{2}-W a^{2}\right], \\
\phi^{\prime \prime}+3 \mathcal{H} \phi^{\prime}-a^{2} \frac{\partial W}{\partial \phi}=0, \\
\chi^{\prime \prime}+3 \mathcal{H} \chi^{\prime}-a^{2} \frac{\partial W}{\partial \chi}=0,
\end{gathered}
$$

where the prime denotes a derivation with respect to the bulk coordinate $w$ and, within this notation, $\mathcal{H}=(\ln a)^{\prime}$. By linearly combining Eqs. (2.6) and (2.7) the following useful pair of equations can be readily obtained:

$$
\begin{aligned}
& \phi^{\prime 2}+\chi^{\prime 2}=\frac{3}{\kappa}\left(\mathcal{H}^{2}-\mathcal{H}^{\prime}\right), \\
& a^{2} W=-\frac{3}{2 \kappa}\left(\mathcal{H}^{\prime}+3 \mathcal{H}^{2}\right) .
\end{aligned}
$$


Needless to say that Eqs. (2.10) and (2.11) are fully equivalent to Eqs. (2.6) and (2.7). In the presence of more than three transverse dimensions, the line element (2.5) can be generalized as

$$
d s^{2}=a^{2}(w)\left[d t^{2}-d x_{1}^{2}-d x_{2}^{2}-\ldots-d x_{d}^{2}-d w^{2}\right],
$$

where the ellipses stand for the $d$ (transverse) spatial coordinates while $w$ still denotes the bulk coordinate. The overall dimensionality of the space-time $D$ will then be given by $D=d+2$. Consequently, in the case of the line element of Eq. (2.12), the analog of Eqs. (2.10) and (2.11) will be

$$
\begin{array}{r}
\phi^{\prime 2}+\chi^{\prime 2}=\frac{d}{\kappa}\left(\mathcal{H}^{2}-\mathcal{H}^{\prime}\right), \\
W(\phi, \chi)=-\frac{d}{2 \kappa a^{2}}\left(\mathcal{H}^{\prime}+d \mathcal{H}^{2}\right) .
\end{array}
$$

Another type of generalization of the system under discussion concerns the addition of quadratic corrections to the Einstein-Hilbert term. In this case the total action is given by

$$
S=S_{\mathrm{EH}}+S_{\mathrm{GB}}
$$

where

$$
S_{\mathrm{GB}}=-\alpha^{\prime} \int d^{5} x \sqrt{|G|} \mathcal{R}_{\mathrm{GB}}^{2}
$$

is the Gauss-Bonnet action ${ }^{2}$ written in terms of the EulerGauss-Bonnet combination, ${ }^{3}$ i.e.

$$
\mathcal{R}_{\mathrm{GB}}^{2}=R_{A B C D} R^{A B C D}-4 R_{A B} R^{A B}+R^{2} .
$$

In four space-time dimensions, the Gauss-Bonnet combination (2.17) is a topological term [42,43] and it coincides with the Euler invariant. This observation implies that, in four space-time dimensions, the contribution of the fourdimensional analog of Eq. (2.16) to the equations of motion can be rearranged in a four-divergence [42].

In more than four space-time dimensions, the contribution of the Gauss-Bonnet combination leads to a ghost-free theory [46]. In particular, the variation of the Gauss-Bonnet action (2.16) brings, at the right-hand side of Eq. (2.1), a new term which can be written as $2 \kappa \alpha^{\prime} \mathcal{Q}_{A B}$ where

\footnotetext{
${ }^{2}$ In Eq. (2.16) $\alpha^{\prime}$ is a constant with dimensions, in natural gravitational units $2 \kappa=1$, of an inverse length. It is practical, at the level of the Einstein-Lanczos equations, to define an effective coupling $\bar{\alpha}=2 \kappa \alpha^{\prime}$ [see below, Eqs. (2.19) and (2.20)].

${ }^{3}$ The Euler-Gauss-Bonnet (or simply Gauss-Bonnet for short) combination [42-50] arises in different higher-dimensional theories and it does also appear in the low-energy string effective action as first correction in the string tension expansion.
}

$$
\begin{aligned}
\mathcal{Q}_{A}^{B}= & \frac{1}{2} \delta_{A}^{B} \mathcal{R}_{\mathrm{GB}}^{2}-2 R R_{A}^{B}+4 R_{A C} R^{C B}+4 R_{C D} R_{A}{ }^{C B D} \\
& -2 R_{A C D E} R^{B C D E}
\end{aligned}
$$

is the so-called Lanczos tensor [42-45] (see also [51] for an interesting review on Gauss-Bonnet gravity and Einstein-Lanczos equations).

The relevant feature to be appreciated is that the inclusion of the contribution provided by Eq. (2.18) generalizes Eqs. (2.10) and (2.11) to a form which is, however, still tractable:

$$
\begin{aligned}
\phi^{\prime 2}+\chi^{\prime 2}= & \frac{3}{\kappa}\left(\mathcal{H}^{2}-\mathcal{H}^{\prime}\right)\left[1-\frac{4 \bar{\alpha}}{a^{2}} \mathcal{H}^{2}\right], \\
W(\phi, \chi)= & -\frac{3}{2 \kappa a^{2}}\left\{\left(\mathcal{H}^{2}-\mathcal{H}^{\prime}\right)+2\left(\mathcal{H}^{2}+\mathcal{H}^{\prime}\right)\right. \\
& \left.\times\left[1-\frac{2 \bar{\alpha}}{a^{2}} \mathcal{H}^{2}\right]\right\},
\end{aligned}
$$

where $\bar{\alpha}=2 \kappa \alpha^{\prime}$. Note that $\bar{\alpha}$ has dimensions of a squared length. Equations (2.19) and (2.20) supplemented by Eqs. (2.8) and (2.9) (which are not affected by the quadratic corrections) form a closed set of equations which will be denoted, for short, as the Einstein-lanczos system.

Finally, as in the case of Einstein-Hilbert gravity, the Einstein-Lanczos Eqs. (2.19) and (2.20) can be further generalized to include $d$ transverse dimensions. In fact Eq. (2.12) implies that Eqs. (2.19) and (2.20) are generalized to

$\phi^{\prime 2}+\chi^{\prime 2}=\frac{d}{\kappa}\left(\mathcal{H}^{2}-\mathcal{H}^{\prime}\right)\left[1-\frac{2 \bar{\alpha}(d-1)(d-2)}{a^{2}} \mathcal{H}^{2}\right]$

$$
\begin{aligned}
W(\phi, \chi)= & -\frac{d}{2 \kappa a^{2}}\left\{\left(\mathcal{H}^{2}-\mathcal{H}^{\prime}\right)+\left[(d-1) \mathcal{H}^{2}\right.\right. \\
& \left.\left.+2 \mathcal{H}^{\prime}\right]\left[1-\frac{\bar{\alpha}(d-1)(d-2)}{a^{2}} \mathcal{H}^{2}\right]\right\} .
\end{aligned}
$$

In the limit when one of the two scalar degrees of freedom is absent it is known that gravitating defects are present and may even lead to realistic warped geometries with a well-defined $\mathrm{AdS}_{5}$ limit for large value of the bulk coordinate. For instance in $[15,16]$ and in [17-20] kink solutions have been presented in the context of EinsteinHilbert gravity and in the presence of either polynomial or generalized sine-gordon potentials. Single kink solutions also may be obtained in Gauss-Bonnet gravity [26-28]. The aim of the subsequent sections will be to show that bound systems of two gravitating defects also may arise naturally in the framework of the present section. Furthermore, particular attention will be given to the case where the five-dimensional geometry possesses the desired $\mathrm{AdS}_{5}$ limit. 


\section{SOLITON-ANTISOLITON SYSTEMS}

Consider, to begin, the case of five-dimensional Einstein-Hilbert gravity with one noncompact extra dimension denoted by $w$. In this case a general ansatz for the soliton-antisoliton system can be written as

$$
\phi(w)=v \sqrt{1+g(w)}, \quad \chi(w)=v \sqrt{1-g(w)},
$$

where $v$ is a dimensionfull constant (i.e. $[v]=L^{-3 / 2}$ ) and $g(w)$ is a dimensionless function of the bulk radius satisfying the following set of properties:

(i) $g(w)$ is a continuous and differentiable function of $w$

(ii) $g(w)$ is a monotonic function of $w$;

(iii) the derivatives of $g(w)$ are also continuous at least up to the second derivative [i.e. $g^{\prime}(w)$ and $g^{\prime \prime}(w)$ are continuous functions of $w]$.

Since the ansatz (3.1) must describe a bound system of a kink and of an antikink it must also be required that

$$
\lim _{w \rightarrow \pm \infty} g(w)= \pm 1 .
$$

The numerical value of the limit is just a reflection of the parametrization (3.1) but it is important, for more general parametrizations, that $g(w)$ goes to a constant for large values of the bulk coordinate. Finally, it is obvious that since $g(w)$ is monotonic, $g^{\prime}(w)$ will never vanish for any finite value of the bulk radius.

Inserting Eq. (3.1) into Eqs. (2.10) and (2.11) a necessary condition on the functional form of $g^{\prime}(w)$ can be obtained:

$$
\left(\frac{d g}{d w}\right)^{2}=\frac{6}{\kappa v^{2}}\left(1-g^{2}\right)\left(\mathcal{H}^{2}-\mathcal{H}^{\prime}\right) .
$$

Once the geometry is specified, Eq. (3.3) allows one to obtain the explicit form of $g(w)$. Then, by fixing the integration constants it is possible to satisfy the conditions required for the existence of a kink-antikink solution.

Consider then, as an example, the simple case when the warp factor is given by

$$
a(w)=\frac{1}{\sqrt{x^{2}+1}}, \quad x=b w,
$$

where $b$ is a dimensionfull constant which will eventually fix the thickness of the configuration. Using Eq. (3.4) into Eq. (3.3) the following differential equation can be readily obtained:

$$
\frac{d g}{d x}= \pm \sqrt{\frac{6}{\kappa v^{2}}} \frac{\sqrt{1-g^{2}}}{x^{2}+1},
$$

whose solution is

$$
g(x)= \pm \frac{x}{\sqrt{x^{2}+1}}, \quad k v^{2}=6 .
$$

The plus sign at the right-hand side of Eq. (3.6) will be conventionally chosen. This choice is, indeed, rather gen- eral and it implies that, according to Eq. (3.1), $\phi(w)$ describes a kink while $\chi(w)$ describes and antikink. In fact, defining the topological charges as

$$
Q_{\phi}=\frac{1}{2 \pi} \int_{-\infty}^{+\infty} \frac{\partial \phi}{\partial w} d w, \quad Q_{\chi}=\frac{1}{2 \pi} \int_{-\infty}^{+\infty} \frac{\partial \chi}{\partial w} d w,
$$

we will have, according to Eqs. (3.1) and (3.6), that $\pi Q_{\phi}=$ $v / \sqrt{2}>0$ and that $\pi Q_{\chi}=-v / \sqrt{2}<0$. If $g(x) \rightarrow-g(x)$, the transformed ansatz is still a solution of Eq. (3.3) [corresponding to the lower sign in Eq. (3.6)] and it implies that $\phi \rightarrow \chi$ and that $\chi \rightarrow \phi$. In other words, if $g \rightarrow-g$ the kink turns into an antikink and vice versa. By choosing the upper sign in Eq. (3.6) we can also write the differential relation appearing in Eq. (3.5) as

$$
\frac{d g}{d x}=\left(1-g^{2}\right)^{3 / 2}
$$

Equations (2.8), (2.9), and (2.11) allow then to compute the specific form of the potential first as a function of $g$ and then as a function of $\phi$ and $\chi$. Using Eqs. (3.4), (3.6), and (3.8) and recalling Eq. (3.1) we will have that, as a function of $g$

$$
\begin{gathered}
W(g)=\frac{3 b^{2}}{2 \kappa}\left(1-5 g^{2}\right), \\
\frac{\partial W}{\partial \phi}(g)=-\frac{b^{2}}{4} v \sqrt{1+g}(1-g)(1+11 g), \\
\frac{\partial W}{\partial \chi}(g)=-\frac{b^{2}}{4} v \sqrt{1-g}(1+g)(1-11 g) .
\end{gathered}
$$

Equations (3.9), (3.10), and (3.11) allow to determine the explicit form of the potential which is

$$
\begin{aligned}
W(\phi, \chi)= & \frac{9 b^{2}}{2 \kappa v^{2}}\left(\phi^{2}+\chi^{2}\right)-\frac{15 b^{2}}{4 \kappa v^{4}}\left(\phi^{4}+\chi^{4}\right) \\
& +\left(\phi^{2}+\chi^{2}-2 v^{2}\right)\left[\frac{11 b^{2}}{16 v^{4}}\left(\phi^{4}+\chi^{4}\right)\right. \\
& \left.-\frac{b^{2}}{2 v^{2}}\left(\phi^{2}+\chi^{2}\right)\right] .
\end{aligned}
$$

It can be explicitly verified, as a useful cross-check, that Eq. (3.12), once inserted into Eqs. (2.6), (2.7), (2.8), and (2.9), solves the system provided the warp factor is given by Eq. (3.4) and the field profiles are the ones of Eq. (3.1).

The obtained result can be generalized to the case of $d$ transverse dimensions [i.e. Eqs. (2.12)]. The calculation follows, in this case, exactly the same steps discussed for the previous five-dimensional example. Less obvious, even if solvable, is the generalization of the obtained results to the situation where the Gauss-Bonnet self-interactions are present. 
Let us show, as a premise, that the ansatz of Eq. (3.1) is not a solution of the five-dimensional Einstein-Lanczos system, i.e. Eqs. (2.19) and (2.20) supplemented by Eqs. (2.8) and (2.9). Equation (3.4) can be generalized to the following form

$$
a(w)=\frac{a_{0}}{\sqrt{x^{2}+1}},
$$

with $a_{0}=2 \sqrt{\bar{\alpha}} b$, as required by the compatibility of Eq. (3.13) with Eq. (2.19). Thus, inserting Eqs. (3.1) and (3.4) into Eq. (2.19) the following differential condition on $g$ can be obtained:

$$
\left(\frac{d g}{d x}\right)^{2}=\beta^{2} \frac{1-g^{2}}{\left(1+x^{2}\right)^{3}}, \quad \beta^{2}=\frac{6}{\kappa v^{2}} .
$$

Now, in the case $\beta=\pi / 2$ the solution of Eq. (3.14) is

$$
g(x)=\sin \left(\frac{\pi}{2} \frac{x}{\sqrt{x^{2}+1}}\right) .
$$

Equation (3.15) certainly satisfies the conditions required for the existence of a kink-antikink solution. However, the compatibility with Eq. (2.20) as well as with Eqs. (2.6) and (2.7) cannot be achieved.

It can be actually shown [41] that the correct ansatz leading to a consistent solution of the EInstein-Lanczos system is given, in this case, by the following expressions:

$$
\begin{aligned}
& \phi(w)=\frac{v}{\sqrt{2}}\left(1+\frac{x}{\sqrt{x^{2}+1}}\right)^{3 / 2}, \\
& \chi(w)=\frac{v}{\sqrt{2}}\left(1-\frac{x}{\sqrt{x^{2}+1}}\right)^{3 / 2},
\end{aligned}
$$

having chosen the warp factor in the form (3.13) with $a_{0}=$ $2 \sqrt{\bar{\alpha}} b$ and with $\kappa v^{2}=4 / 3$. In this case the potential $W(\phi, \chi)$ can be written as

$$
\begin{aligned}
W(\tilde{\phi}, \tilde{\chi})= & \frac{3 v^{2}}{2 \bar{\alpha}}\left(\tilde{\phi}^{2}+\tilde{\chi}^{2}\right)^{2}-\frac{3 v^{2}}{\bar{\alpha}}\left(\tilde{\phi}^{2}+\tilde{\chi}^{2}\right)+\frac{15}{16} \frac{v^{2}}{\bar{\alpha}} \\
& +\frac{7}{2} \frac{v^{2}}{\bar{\alpha}}\left(|\tilde{\phi}|^{2 / 3}+|\tilde{\chi}|^{2 / 3}-1\right)\left(1-\tilde{\phi}^{2}-\tilde{\chi}^{2}\right)^{2},
\end{aligned}
$$

where, for notational convenience, the canonical fields $\tilde{\phi}$ and $\tilde{\chi}$ have been introduced (note that $\phi=2 v \tilde{\phi}$ and $\chi=$ $2 v \tilde{\chi})$. The solution expressed by Eqs. (3.16) and (3.17) is qualitatively similar to the one of Eq. (3.1) but it is, at the same time, mathematically different. Even if the geometry is given, in both cases, by a warp factor that tends to $\mathrm{AdS}_{5}$ for large absolute value of the bulk coordinate, the $\mathrm{AdS}_{5}$ radius is different in the two cases given, respectively, by Eqs. (3.4) and (3.13) the difference being given by $a_{0}=$ $2 \sqrt{\bar{\alpha}} b$. Finally, the solution (3.16) and (3.17) holds for $\phi>$ 0 and $\chi>0$. However, also $\phi \rightarrow-\phi$ and $\chi \rightarrow-\chi$ is a solution of the system as it is clear from the absolute values appearing in Eq. (3.18).
In Fig. 1 the kink-antikink solutions are illustrated for the cases of the Einstein-Hilbert kink [see Eqs. (3.1) and (3.6)] and of the Gauss-Bonnet kink [see Eqs. (3.16) and (3.17)]. In Fig. 1 the dashed line denotes the antikink while the full line illustrates the kink. It should be stressed that the main objective of this paper is not to find single gravitating kinks (or single antikinks) but rather to find solutions where two field configurations are simultaneously present with two opposite topological charges.

While the solutions reported in Fig. 1 are interesting by themselves, it is worth stressing that, in the present section, a rather general method for constructing multidefects solutions has been proposed. The same method will also be exploited in the forthcoming sections in order to generalize the present considerations to the case of bound systems of topological and nontopological solitons.

It should be stressed that the solutions discussed in the present section can be generalized to the case where there are, generically, $d$ transverse dimensions. In fact, owing to the form of Eqs. (2.13) and (2.14) and of Eqs. (2.21) and (2.22), the same analytical steps can be successfully carried on.

A final remark is in order concerning the interplay of the obtained solutions and the case of $(3+1)$-dimensional domain walls. In $(3+1)$ dimensions, Derrick's theorem implies that static domain walls cannot exist in a theory with scalar fields as the one considered in this paper. Furthermore, Derrick's theorem also implies that the same result would hold in five (space-time) dimensions. We recall here that the resolution of this impasse is that Derrick's theorem holds in the absence of gravitational interactions, i.e. in the case where the geometry is Minkowskian also in higher dimensions. In the present

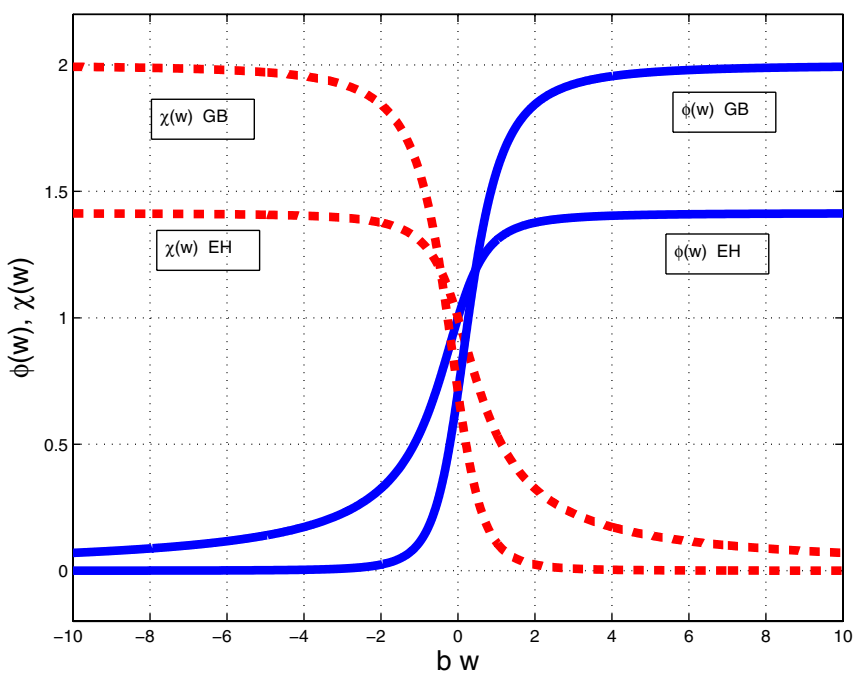

FIG. 1 (color online). The Einstein-Hilbert (EH) and the Gauss-Bonnet (GB) kink-antikink systems are illustrated, by fixing, conventionally, $v=1$ in natural gravitational units $2 \kappa=$ 1. The full lines denote the kink profiles while the dashed lines denote the antikink profiles. 

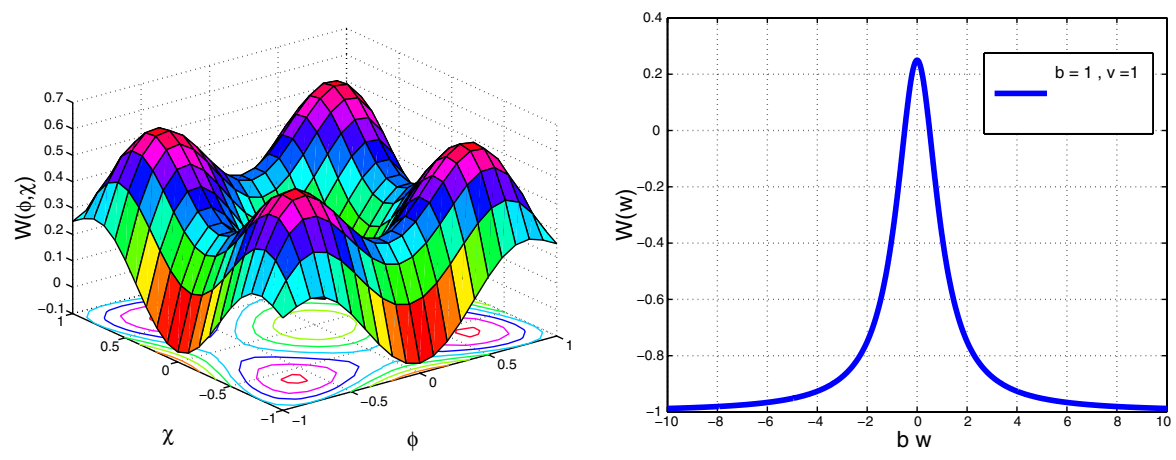

FIG. 2 (color online). The potential obtained in Eq. (3.12) is illustrated for the case $b=v=1$.

case gravity is an essential ingredient. It indeed can be seen that the $\mathrm{AdS}_{5}$ nature of the geometry implies that, effectively, the potential of the solutions discussed in the present section, is equivalent to a negative cosmological constant. This point is illustrated in Fig. 2 where the potential derived in Eq. (3.12) is plotted. In the plot at the left the potential is reported as a function of $\phi$ and $\chi$. In the plot at the right the potential is reported as a function of the bulk coordinate $w$. The plot at the right is obtained by substituting in the potential the actual solutions for $\phi$ and $\chi$. For the sake of simplicity we have set, in Fig. $2, b=v=1$. It is clear that the potential gets negative playing, therefore, the role of a negative cosmological term.

\section{TOPOLOGICAL AND NONTOPOLOGICAL SOLITONS}

In the present section a general class of solutions containing pairs of defects will be presented. The new feature of this class is that, for some values of a discrete parameter, the solutions describe a system containing simultaneously a topological and a nontopological defect. In the same class of solutions, it is also possible to find configurations that can be interpreted as a classically bound system of two nontopological profiles.

Consider, for this purpose, the following form of the warp factor:

$$
a(w)=\left[x^{2 \nu}+1\right]^{-(1 / 2 \nu)}, \quad x=b w,
$$

where $\nu$ is a natural number. Following a similar procedure of the one presented in the previous section consider also the following ansatz for the field profiles:

$$
\begin{aligned}
& \phi(w)=v\left\{[1+h(w)]^{3 / 2}+[1-h(w)]^{3 / 2}\right\}, \\
& \chi(w)=v\left\{[1+h(w)]^{3 / 2}-[1-h(w)]^{3 / 2}\right\},
\end{aligned}
$$

where $h(w)$ is a continuous function sharing, for a topological soliton, exactly the same properties of $g(w)$. In particular, for a topological soliton, it will be required that

$$
\lim _{w \rightarrow \pm \infty} h(w)= \pm 1
$$

The analog of Eq. (4.4) in the case of a nontopological profile will instead be

$$
\lim _{|w| \rightarrow \infty} h(w)= \pm 1
$$

In spite of a superficial similarity, Eqs. (4.4) and (4.5) imply, indeed, different boundary conditions. In the case of Eq. (4.4) the function $h(w)$ goes either to -1 or to +1 provided $w$ goes, respectively, either to $-\infty$ or to $+\infty$. For the boundary conditions expressed by Eq. (4.5), the function $h(w)$ goes to the same value as soon as $|w| \rightarrow \infty$. This value can be either +1 or -1 since, as it will be discussed in a moment, the change $h \rightarrow-h$ still leads to a viable solution. It is important to remark that, as a consequence of the different boundary conditions at infinity, the function $h(w)$ is either monotonic (topological profile) or nonmonotonic (nontopological profile). In this sense $h(w)$ shares exactly the same properties of $g(w)$ only in the case of topological configurations.

Working in the framework of five-dimensional EinsteinHilbert gravity, Eq. (2.10) allows the determination of $h(w)$ whose functional form must obey the following differential condition:

$$
\left(\frac{d h}{d x}\right)^{2}=\left(\frac{2 \nu-1}{3 \kappa v^{2}}\right) \frac{x^{2 \nu-2}}{x^{2 \nu}+1}
$$

whose solution is

$$
h(x)=\frac{2}{\pi} \arctan \left(x^{\nu}\right), \quad \kappa v^{2}=\frac{\pi^{2}}{12} \frac{2 \nu-1}{\nu^{2}} .
$$

Equation (4.7) implies that if $\nu$ is an odd integer, $h(x)$ will necessarily interpolate between -1 and +1 [as required in the first set of boundary conditions written in Eq. (4.4)]. If, on the contrary, $\nu$ is an even integer, $h(x)$ will always go to +1 as soon as $|x| \rightarrow \infty$ [as required in the second set of boundary conditions written in Eq. (4.5)]. Furthermore, in the case of even $\nu, h(x)$ will have a global minimum in $x=$ 0 . As already anticipated in the previous paragraph, the form of the differential condition (4.6) implies that if $h(x)$ is a solution, also $-h(x)$ will be a solution. If $h \rightarrow-h$, Eqs. (4.2) and (4.3) imply that $\phi \rightarrow \phi$ and $\chi \rightarrow-\chi$. 
The difference between the solutions with odd $\nu$ and the ones with even $\nu$ implies also different physical properties which will be one of the themes of the forthcoming discussion. The potential satisfying Eqs. (2.8), (2.9), and (2.11) [or, equivalently, Eqs. (2.6), (2.7), (2.8), and (2.9)] can be written as

$$
\begin{aligned}
W(\phi, \chi)= & \frac{3 b^{2}}{2 \kappa}\left[\sin ^{2} \sigma\right]^{(\nu-1) / \nu}\left[(2 \nu-1)-(2 \nu+3) \sin ^{2} \sigma\right] \\
& +\left[|\tilde{\phi}+\tilde{\chi}|^{2 / 3}+|\tilde{\phi}-\tilde{\chi}|^{2 / 3}-2\right] \mathcal{A}
\end{aligned}
$$

where, as usual, $\phi=2 v \tilde{\phi}$ and $\chi=2 v \tilde{\chi}$. The functions $\sigma(\tilde{\phi}, \tilde{\chi})$ and $\mathcal{A}(\tilde{\phi}, \tilde{\chi})$ appearing in Eq. (4.8) are defined, respectively, as

$$
\begin{aligned}
\mathcal{A}(\tilde{\phi}, \tilde{\chi})= & \frac{18 b^{2} v^{2} \nu}{\pi^{2}} \cos \sigma\left\{\sigma\left[\sin ^{2} \sigma\right]^{(\nu-2) / 2 \nu}[(\nu-1)\right. \\
& \left.\left.-(2 \nu+3) \sin ^{2} \sigma\right]+\frac{\nu}{2} \cos \sigma\left[\sin ^{2} \sigma\right]^{(\nu-1) / \nu}\right\},
\end{aligned}
$$

$$
\sigma(\tilde{\phi}, \tilde{\chi})=\frac{\pi}{4}\left[|\tilde{\phi}+\tilde{\chi}|^{2 / 3}-|\tilde{\phi}-\tilde{\chi}|^{2 / 3}\right] .
$$

Let us now scrutinize the physical properties of the obtained solution. From Eq. (4.1), (both for even and odd values of $\nu$ ), the warp factor always tend to $\mathrm{AdS}_{5}$ for $|w| \rightarrow$ $\infty$ i.e.

$$
\lim _{w \rightarrow \pm \infty} a(w)=\frac{1}{b|w|} .
$$

The limit (4.11) reproduces the asymptotic behavior of the warp factors considered in Eqs. (3.4) and (3.13). Furthermore, in the cases of Eqs. (3.4), (3.13), and (4.1) the geometry is always regular since all the curvature invariants are never divergent at finite values of the bulk coordinate and they go to a constant in the limit $|w| \rightarrow \infty$ as implied by the $\mathrm{AdS}_{5}$ limit of the warp factors.

As previously argued in connection with the properties of $h(x)$ the features of the solutions differ depending on the even or odd values of $\nu$. Consider the first few odd values of $\nu$, i.e. $\nu=1,3,5 \ldots$ The analysis of these situations will allow one to gain intuition on the more general case. In Fig. 3 the behavior of $\phi(w)$ (left panel) and of $\chi(w)$ (plot at the right) is illustrated as a function of the dimensionless bulk coordinate $x=b w$ for $\nu=1$ and $\nu=3$. Even if $\phi$ and $\chi$ are solutions, for a given value of $\nu$, of the same system of equations, the properties of the obtained defects are different. In particular, for odd values of $\nu, \phi$ always describes a nontopological profile. More specifically, Eq. (4.2) implies

$$
\lim _{w \rightarrow \pm \infty} \phi(w)=2 \sqrt{2} v .
$$

Note that in Fig. 3 (as well in all the other figures) the value of $v$ has been set to 1 in natural gravitational units $2 \kappa=1$.

For the same odd values of $\nu$, the corresponding solution for $\chi(w)$ describes instead a topological soliton. In this case (see Fig. 3, right plot) the field profile interpolates between $-2^{3 / 2} v$ and $+2^{3 / 2} v$ :

$$
\lim _{w \rightarrow-\infty} \chi(w)=-2 \sqrt{2} v, \quad \lim _{w \rightarrow+\infty} \chi(w)=+2 \sqrt{2} v .
$$

In the case $\nu=3$ the solution also exhibits a short plateau centered around $w=0$. Larger (odd) values of $\nu$ lead to profiles which are qualitatively similar to the ones discussed in the cases of Fig. 3. It is therefore legitimate to conclude that, for odd values of $\nu$, the solution defined by Eqs. (4.2), (4.3), and (4.7) always describe a bound system of a topological soliton (the $\chi$ field) and of a nontopological defect (the $\phi$ field). Similar solutions also arise in $(1+$ 1)-dimensional field theories with appropriate nonlinear potentials, and, in that context, they have been named "trapping bags" $[1,3,12]$ since, in our language, the $\chi$ field is "trapped" in the "bag" provided by the $\phi$ profile.

From Eq. (4.6) it follows that if $h(x)$ is a solution, also $-h(x)$ will be a solution. If $h \rightarrow-h$, then, from Eqs. (4.2) and (4.3), the role of $\phi$ and $\chi$ will be interchanged. In

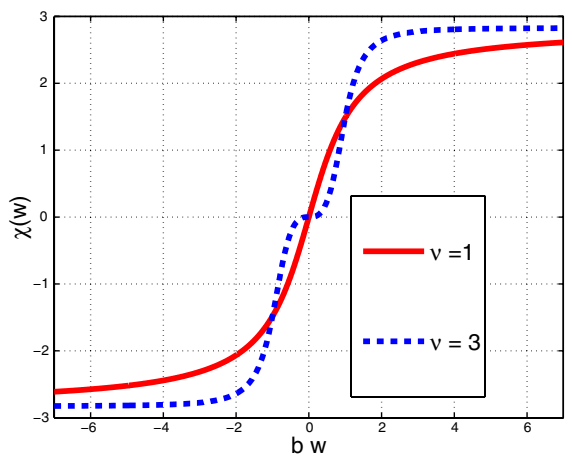

FIG. 3 (color online). The trapping bag solutions arising in the case of odd values of $\nu$ are illustrated for few typical cases and by choosing, as in Fig. 1, $v=1$. The full lines refer to the case $\nu=1$ while the dashed lines to the case $\nu=3$. It should be appreciated that the topological (plot at the right) and the nontopological (plot at the left) profiles are both simultaneously present for each odd value of $\nu$. 

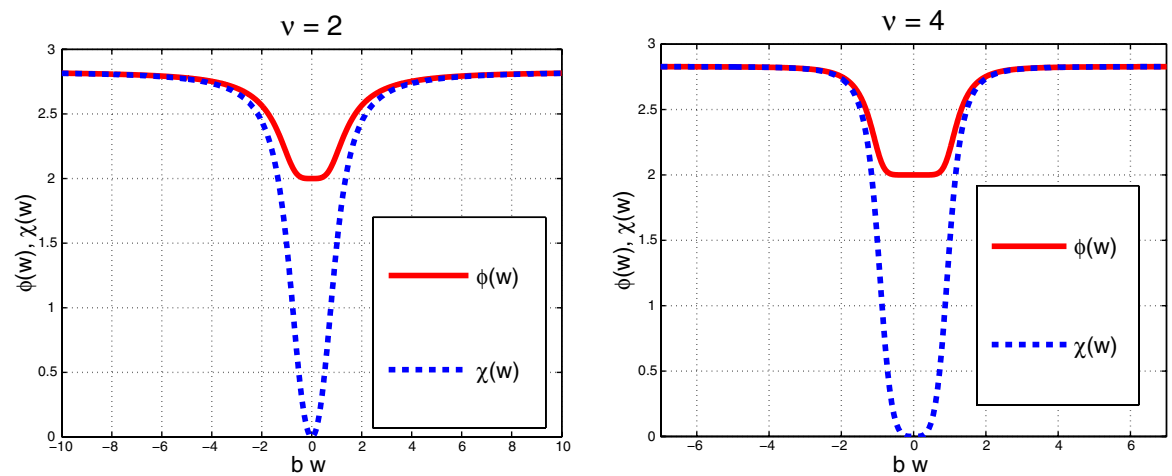

FIG. 4 (color online). The bag-bag solutions arising in the case of even values of $\nu$ are illustrated for $\nu=2$ (left plot) and for $\nu=4$ (right plot). The value of $v$ is, as usual, set to 1 in natural gravitational units.

particular, in the case of odd $\nu, h \rightarrow-h$ would imply that the topological profile becomes nontopological and vice versa. Let us now consider the case when $\nu$ is an even integer, i.e. $\nu=2,4,6, \ldots$ In Fig. 4 the first two relevant values of $\nu$ are illustrated. In this situation it is not difficult to get convinced, by looking at Eqs. (4.2), (4.3), and (4.7) that $\phi(w)$ and $\chi(w)$ both describe nontopological profiles and that the overall solution is therefore a bound system of two bags, according to the terminology previously employed in the present section. This statement can be explicitly verified since, in the case of even $\nu$, it holds that

$$
\lim _{w \rightarrow \pm \infty} \phi(w)=\lim _{w \rightarrow \pm \infty} \chi(w)=2 \sqrt{2} v .
$$

It can be argued from Fig. 4 that as $\nu$ increases the width of the bag also increases. Moreover, both $\phi$ and $\chi$ have a global minimum for $w=0$. However, while $\chi(0)=0$, $\phi(0)=2 v$. The configurations arising in the case of even values of $\nu$ illustrate the system formed of two bag profiles (i.e. bag-bag solutions).

\section{ZERO MODES FOR MULTIDEFECTS SYSTEMS}

In the following the techniques for the analysis of the zero modes in the case of gravitating multidefects will be presented. The treatment followed in the present section generalizes the gauge-invariant approach to the fluctuations of single defects developed in $[19,20]$. The main differences of the discussion resides in the analysis of the scalar modes. In the case of a single gravitating defect in five-dimensional warped geometries it was shown that

(i) the scalar modes of the configurations can be found by solving a single second-order (linear) differential equation for a gauge-invariant variable which is the combination of the fluctuations of the geometry and of the fluctuation of the defect;

(ii) this gauge-invariant degree of freedom is indeed the canonical normal mode of the system, as it was demonstrated [37] by diagonalizing the full second-order action for the scalar modes of the geometry coupled with the fluctuations of the defect; (iii) the zero mode (i.e. the lowest mass eigenstate) was shown not to be normalizable.

The introduction of a further scalar degree of freedom entails necessarily the need of a second gauge-invariant variable. While the generalization of the tensor and vector problem will be swiftly discussed just for completeness, the treatment of the scalar modes is qualitatively different with respect to single-defect configurations.

\section{A. General considerations}

Denoting by $\delta \phi$ and $\delta \chi$ the first-order perturbations of $\phi$ and $\chi$, the scalar degrees of freedom will be given by their background values supplemented by the appropriate fluctuations:

$$
\phi \rightarrow \phi(w)+\delta \phi\left(x^{\mu}, w\right), \quad \chi \rightarrow \phi(w)+\delta \chi\left(x^{\mu}, w\right) .
$$

By taking the trace of Eq. (2.1) we get

$$
R_{A B}=\kappa\left(T_{A B}-\frac{T}{3} G_{A B}\right), \quad T_{A}^{A}=T,
$$

which is particularly useful in perturbation theory since it gives automatically all the relevant equations avoiding the calculation of the first-order fluctuation of the Ricci scalar. Therefore, by taking the first-order fluctuation of Eq. (5.2) and by recalling Eq. (2.4) we will have

$$
\begin{aligned}
\delta R_{A B}= & \kappa\left[\partial_{A} \delta \phi \partial_{B} \phi+\partial_{A} \phi \partial_{B} \delta \phi+\partial_{A} \delta \chi \partial_{B} \chi\right. \\
& \left.+\partial_{A} \chi \partial_{B} \delta \chi\right]-\frac{2 \kappa}{3} W(\phi, \chi) \delta G_{A B} \\
& -\frac{2 \kappa}{3} \bar{G}_{A B}\left(\frac{\partial W}{\partial \phi} \delta \phi+\frac{\partial W}{\partial \chi} \delta \chi\right),
\end{aligned}
$$

where $\bar{G}_{A B}$ denotes the background value of the metric tensor while $\delta G_{A B}$ and $\delta R_{A B}$ denote, respectively, the fluctuation of the five-dimensional metric and the fluctuation of the five-dimensional Ricci tensor.

With similar notations, the first-order version of Eqs. (2.2) and (2.3) can be written by recalling that, when acting on a scalar degree of freedom, $\nabla_{A} \nabla_{B}=\partial_{A} \partial_{B}-$ $\Gamma_{A B}^{C} \partial_{C}$ where $\Gamma_{A B}^{C}$ is the five-dimensional Christoffel connection. With this specification in mind we will have that 


$$
\begin{array}{r}
\delta G^{A B}\left[\partial_{A} \partial_{B} \phi-\bar{\Gamma}_{A B}^{C} \partial_{C} \phi\right]+\bar{G}^{A B}\left[\partial_{A} \partial_{B} \delta \phi-\delta \Gamma_{A B}^{C} \partial_{C} \phi\right. \\
\left.-\bar{\Gamma}_{A B}^{C} \partial_{C} \delta \phi\right]+\frac{\partial^{2} W}{\partial \phi^{2}} \delta \phi+\frac{\partial^{2} W}{\partial \phi \partial \chi} \delta \chi=0, \\
\delta G^{A B}\left[\partial_{A} \partial_{B} \chi-\bar{\Gamma}_{A B}^{C} \partial_{C} \chi\right]+\bar{G}^{A B}\left[\partial_{A} \partial_{B} \delta \chi-\delta \Gamma_{A B}^{C} \partial_{C} \chi\right. \\
\left.-\bar{\Gamma}_{A B}^{C} \partial_{C} \delta \chi\right]+\frac{\partial^{2} W}{\partial \chi^{2}} \delta \chi+\frac{\partial^{2} W}{\partial \phi \partial \chi} \delta \phi=0 .
\end{array}
$$

In Eqs. (5.4) and (5.5), $\bar{\Gamma}_{A B}^{C}$ and $\delta \Gamma_{A B}^{C}$ denote, respectively, the background Christoffel and their first-order fluctuations.

As usual [19], the 15 degrees of freedom constituting $\delta G_{A B}$ in five dimensions ${ }^{4}$ can be separated, to first-order, into tensor, vector, and scalar modes. In particular, this decomposition amounts to parametrize the various perturbed components of the metric tensor as

$$
\begin{gathered}
\delta G_{\mu \nu}=2 a^{2} h_{\mu \nu}+a^{2}\left(\partial_{\mu} f_{\nu}+\partial_{\nu} f_{\mu}\right)+2 a^{2} \eta_{\mu \nu} \psi \\
+2 a^{2} \partial_{\mu} \partial_{\nu} E, \\
\delta G_{\mu w}=a^{2}\left(D_{\mu}+\partial_{\mu} C\right), \\
\delta G_{w w}=2 a^{2} \xi,
\end{gathered}
$$

where the Lorentz indices run over the four space-time dimensions. The tensor modes, i.e. $h_{\mu \nu}$, are, by definition, traceless and divergenceless, i.e. $\partial_{\mu} h_{\nu}^{\mu}=h_{\mu}^{\mu}$. Furthermore, it can be shown that, under infinitesimal coordinate transformations $h_{\mu \nu}$ is invariant. The invariance of a given fluctuations with respect to infinitesimal coordinate transformations will be referred to as gaugeinvariance since the group of infinitesimal diffeomorphisms is, effectively, the gauge group of gravitation.

The vector modes are parametrized by two divergenceless vectors i.e. $f_{\mu}$ and $D_{\mu}$ subjected to the conditions $\partial_{\mu} D^{\mu}=0$ and $\partial_{\mu} f^{\mu}=0$. Overall there are 6 degrees of freedom.

Finally, the scalar modes are parametrized in terms of the four Lorentz scalars $\psi, \xi, E$, and $C$ whose evolution equations are coupled with the fluctuations of the multidefect, i.e. $\delta \phi$ and $\delta \chi$.

\section{B. Tensor zero mode}

The evolution equation of the tensor modes is given by

$$
h_{\mu \nu}^{\prime \prime}+3 \mathcal{H} h_{\mu \nu}^{\prime}-\square h_{\mu \nu}=0,
$$

where $\square=\eta^{\alpha \beta} \partial_{\alpha} \partial_{\beta}$. The zero mode of the tensor fluctuations is localized in all the solutions presented in the previous sections owing to the quasi-AdS $S_{5}$ nature of the

\footnotetext{
${ }^{4}$ While it is rather simple to discuss the same decomposition in the case of $d$ transverse dimensions [52], we will be concerned mainly here with the case $d=3$.
}

geometry. Defining, in fact, the rescaled variable $\mu_{\mu \nu}=$ $a^{3 / 2} h_{\mu \nu}$, Eq. (5.9) becomes

$$
\mu_{\mu \nu}^{\prime \prime}-\square \mu_{\mu \nu}-\frac{\left(a^{3 / 2}\right)^{\prime \prime}}{a^{3 / 2}} \mu_{\mu \nu}=0 .
$$

Note that $\mu_{\mu \nu}$ is the variable which appears canonically normalized in the (second-order) action. Neglecting the Laplacian, the solution of Eq. (5.10) which is normalizable is given by $\mu_{\mu \nu}=c_{\mu \nu} a^{3 / 2}$ where $c_{\mu \nu}$ does not depend on $w$ and $\mu_{0}(w)=a^{3 / 2}(w)$. Thus, the tensor zero mode is normalizable provided the integral

$$
\int_{-\infty}^{+\infty}\left|\mu_{0}(w)\right|^{2} d w=\int_{-\infty}^{+\infty} a^{3}(w) d w
$$

is finite. This normalizability condition is satisfied by all the explicit forms of the warp factors discussed so far in the present paper and, in particular, by Eqs. (3.4) and (4.1). In particular, it turns out that

$$
\begin{aligned}
& \int_{-\infty}^{\infty} \frac{d w}{\left[1+(b w)^{2}\right]^{3 / 2}}=\frac{2}{b} \\
& \int_{-\infty}^{\infty} \frac{d w}{\left[1+(b w)^{2 \nu}\right]^{3 / 2 \nu}}=\frac{2}{b} \frac{\Gamma\left(1+\frac{1}{2 \nu}\right) \Gamma\left(\frac{1}{\nu}\right)}{\Gamma\left(\frac{3}{2 \nu}\right)},
\end{aligned}
$$

where the result of the second integral in (5.12) is obtained under the assumption that $\nu$ is a natural number, i.e. the same assumption for which the solutions reported in Sec. IV are defined. The finiteness of the integral appearing in Eq. (5.11) is also required by the finiteness of the fourdimensional Planck mass

$$
M_{\mathrm{P}}^{2}=M_{5}^{3} \int_{-\infty}^{+\infty} a^{3}(w) d w .
$$

\section{Vector zero mode}

For infinitesimal coordinate transformations

$$
x^{A} \rightarrow \tilde{x}^{A}=x^{A}+\epsilon^{A}, \quad \epsilon_{A}=a^{2}(w)\left(\epsilon_{\mu},-\epsilon_{w}\right),
$$

the scalar and vector modes of the geometry are transformed. In particular, by isolating the divergenceless part of $\epsilon_{\mu}$ as

$$
\epsilon_{\mu}=\partial_{\mu} \epsilon+\zeta_{\mu}, \quad \partial_{\mu} \zeta^{\mu}=0,
$$

the gauge variation of the vector modes is

$$
f_{\mu} \rightarrow \tilde{f}_{\mu}=f_{\mu}-\zeta_{\mu}, \quad D_{\mu} \rightarrow \tilde{D}_{\mu}=D_{\mu}-\zeta_{\mu}^{\prime} .
$$

From Eq. (5.16) it is immediate to ascertain the gaugeinvariant vector mode which is given by

$$
V_{\mu}=D_{\mu}-f_{\mu}^{\prime} \text {. }
$$

From the explicit form of Eq. (5.3) it is possible to obtain, after some algebra, that the zero mode of $V_{\mu}$ obeys the 
following equation:

$$
\mathcal{V}_{\mu}^{\prime}+\frac{3}{2} \mathcal{H} \mathcal{V}_{\mu}=0, \quad \mathcal{V}_{\mu}=a^{3 / 2} V_{\mu}
$$

From Eq. (5.18) the normalizability of the vector zero mode implies the convergence of the following integral:

$$
\int_{-\infty}^{\infty} \frac{d w}{a^{3}(w)}
$$

It is clear that the normalizability condition implied by Eq. (5.19) is, somehow, opposite to the one arising from Eqs. (5.11) and (5.13). The conclusion is, therefore, exactly the same as one would get in the case of single defects: if the graviton is normalizable, then the Planck mass is finite and the graviphoton is not normalizable. It should be remarked that this conclusion is typical of five-dimensional warped geometries. However, in even higher dimensions the situation may change. In particular, six-dimensional examples $[53,54]$ seem to suggest that some vector degrees of freedom may indeed be localized (see also [55] for warped cosmological backgrounds in six dimensions).

\section{Scalar zero modes}

While the discussion of the tensor and vector modes of the geometry mirrors completely the case of single defects, the scalar mode presents relevant qualitative differences.

The scalar modes of the geometry are not gauge invariant and are, in some sense, the most relevant ones since they are coupled with the fluctuations of the multidefect configurations. More specifically, for the gauge transformation written in Eqs. (5.14) and (5.15) the scalar fluctuations of the geometry change as

$$
\begin{gathered}
\psi \rightarrow \tilde{\psi}=\psi-\mathcal{H} \epsilon_{w}, \\
\xi \rightarrow \tilde{\xi}=\xi+\mathcal{H} \epsilon_{w}+\epsilon_{w}^{\prime}, \\
C \rightarrow \tilde{C}=C-\epsilon^{\prime}+\epsilon_{w}, \\
E \rightarrow \tilde{E}=E-\epsilon .
\end{gathered}
$$

To these transformations, the gauge variations of the fluctuations of the defects must be added and they are

$$
\begin{gathered}
\delta \phi \rightarrow \tilde{\delta} \phi=\delta \phi-\phi^{\prime} \epsilon_{w}, \\
\delta \chi \rightarrow \tilde{\delta} \chi=\delta \chi-\chi^{\prime} \epsilon_{w} .
\end{gathered}
$$

From Eqs. (5.20), (5.21), (5.22), (5.23), (5.24), and (5.25) it is clear that while gauge transformations involving $\zeta_{\mu}$ preserve the vector nature of the fluctuation, the transformations involving $\epsilon$ and $\epsilon_{w}$ preserve the scalar nature of the fluctuation.

It is possible, in the context of the scalar modes, to define a set of gauge-invariant variables which are, in their simplest incarnation:

$$
\begin{gathered}
\Psi=\psi-\mathcal{H}\left(E^{\prime}-C\right), \\
\Xi=\xi-\frac{1}{a}\left[a\left(C-E^{\prime}\right)\right]^{\prime}, \\
X=\delta \chi+\chi^{\prime}\left(C-E^{\prime}\right), \\
\Phi=\delta \phi+\phi^{\prime}\left(C-E^{\prime}\right) .
\end{gathered}
$$

In terms of the fluctuations defined in Eqs. (5.26), (5.27), (5.28), and (5.29) the evolution equations of the scalar problem can be written in fully gauge-invariant terms. In particular, from the diagonal components of Eq. (5.3), i.e. the components $(\mu \mu)$ and $(w w)$, the following pair of equations can be obtained:

$$
\begin{aligned}
\square \Xi & +4 \Psi^{\prime \prime}+4 \mathcal{H}\left(\Psi^{\prime}+\Xi^{\prime}\right)-\frac{4}{3} \kappa W a^{2} \Xi \\
& +2 \kappa\left[\phi^{\prime} \Phi^{\prime}+\chi^{\prime} X^{\prime}\right]+\frac{2}{3} \kappa a^{2}\left[\frac{\partial W}{\partial \phi} \Phi+\frac{\partial W}{\partial \chi} X\right]=0,
\end{aligned}
$$

$$
\begin{gathered}
\Psi^{\prime \prime}+7 \mathcal{H} \Psi^{\prime}+\mathcal{H} \Xi^{\prime}-\square \Psi+2\left(\mathcal{H}^{\prime}+3 \mathcal{H}^{2}\right) \Xi \\
+\frac{2}{3} \kappa a^{2}\left[\frac{\partial W}{\partial \phi} \Phi+\frac{\partial W}{\partial \chi} X\right]=0 .
\end{gathered}
$$

From the off-diagonal elements of Eq. (5.3), i.e. $(\mu \neq \nu)$ and $(\mu w)$ we do get the following two conditions:

$$
\begin{gathered}
\partial_{\mu} \partial_{\nu}[\Xi-2 \Psi]=0, \\
3\left(\Psi^{\prime}+\mathcal{H} \Xi\right)+\kappa\left(\phi^{\prime} \Phi+\chi^{\prime} X\right)=0 .
\end{gathered}
$$

Finally, from Eqs. (5.4) and (5.5) we get the evolution equations for $\Phi$ and $X$, i.e. the gauge-invvariant fluctuations of the defects:

$$
\begin{aligned}
\Phi^{\prime \prime}+ & 3 \mathcal{H} \Phi^{\prime}-\square \Phi-\frac{\partial^{2} W}{\partial \varphi^{2}} a^{2} \Phi-\frac{\partial^{2} W}{\partial \phi \partial \chi} a^{2} X \\
& +\phi^{\prime}\left[4 \Psi^{\prime}+\Xi^{\prime}\right]+2\left(\phi^{\prime \prime}+3 \mathcal{H} \phi^{\prime}\right) \Xi=0, \\
X^{\prime \prime}+ & 3 \mathcal{H} X^{\prime}-\square X-\frac{\partial^{2} W}{\partial \varphi^{2}} a^{2} X-\frac{\partial^{2} W}{\partial \phi \partial \chi} a^{2} \Phi \\
& +\chi^{\prime}\left[4 \Psi^{\prime}+\Xi^{\prime}\right]+2\left(\chi^{\prime \prime}+3 \mathcal{H} \chi^{\prime}\right) \Xi=0 .
\end{aligned}
$$

By subtracting Eqs. (5.30) and (5.31) and by using the constraint $\Xi=2 \Psi$ the following equation can be obtained:

$$
\Psi^{\prime \prime}+\mathcal{H} \Psi^{\prime}+\square \Psi=-\frac{2 \kappa}{3}\left[\phi^{\prime} \Phi^{\prime}+\chi^{\prime} X^{\prime}\right] .
$$

Moreover, using, again, the condition stemming from Eq. (5.32) it is easy to reduce Eqs. (5.34) and (5.35) to the following (more tractable) form: 


$$
\begin{aligned}
\Phi^{\prime \prime}+3 \mathcal{H} \Phi^{\prime}- & \square \Phi-\frac{\partial^{2} W}{\partial \varphi^{2}} a^{2} \Phi-\frac{\partial^{2} W}{\partial \phi \partial \chi} a^{2} X \\
+ & 6 \Psi^{\prime} \phi^{\prime}+4\left(\phi^{\prime \prime}+3 \mathcal{H} \phi^{\prime}\right) \Psi=0, \\
X^{\prime \prime}+3 \mathcal{H} X^{\prime}- & \square X-\frac{\partial^{2} W}{\partial \varphi^{2}} a^{2} X-\frac{\partial^{2} W}{\partial \phi \partial \chi} a^{2} \Phi \\
& +6 \chi^{\prime} \Psi^{\prime}+4\left(\chi^{\prime \prime}+3 \mathcal{H} \chi^{\prime}\right) \Psi=0 .
\end{aligned}
$$

In the presence of a single defect, the system describing the scalar modes of the geometry and of the sources can be put in a diagonal form by introducing the appropriately normal mode of the scalar system which is linear combination (with background-dependent coefficients) of the fluctuation of the geometry and of the fluctuations of the defect [37]. If two (or more) defects are simultaneously present this strategy can be generalized with important qualitative differences. Let us therefore define the following pair of variables:

$$
\begin{aligned}
& \mathcal{G}=a^{3 / 2} \Phi-z_{\phi} \Psi, \\
& \mathcal{F}=a^{3 / 2} X-z_{\chi} \Psi,
\end{aligned}
$$

where

$$
z_{\phi}=\frac{a^{3 / 2} \phi^{\prime}}{\mathcal{H}}, \quad z_{\chi}=\frac{a^{3 / 2} \chi^{\prime}}{\mathcal{H}} .
$$

Equations (5.39) and (5.40) can be used to eliminate $\Phi$ and $X$ from Eqs. (5.37) and (5.38). The same procedure can be adopted in Eq. (5.36): the dependence upon $\Phi$ and $X$ appearing at the right-hand side of Eq. (5.36) can be eliminated in favor of $\mathcal{G}$ and $\mathcal{F}$. The obtained equation can be used to eliminate the dependence on $\Psi$ arising in Eqs. (5.37) and (5.38). The net result of this procedure is that the evolution equations for $\mathcal{G}$ and $\mathcal{F}$ is given by

$$
\begin{gathered}
\mathcal{G}^{\prime \prime}-\square \mathcal{G}-\mathcal{M}_{G \mathcal{G}} \mathcal{G}-\mathcal{M}_{\mathcal{G}} \mathcal{F}=0, \\
\mathcal{F}^{\prime \prime}-\square \mathcal{F}-\mathcal{M}_{\mathcal{F} \mathcal{F}} \mathcal{F}-\mathcal{M}_{\mathcal{F} G} \mathcal{G}=0,
\end{gathered}
$$

where

$$
\begin{aligned}
\mathcal{M}_{G G}= & \frac{\left(a^{3 / 2}\right)^{\prime \prime}}{a^{3 / 2}}+a^{2} \frac{\partial^{2} W}{\partial \phi^{2}}-\frac{2 \kappa}{3 \mathcal{H}^{2}} \mathcal{H}^{\prime} \phi^{\prime 2}+\frac{4}{3} \kappa \frac{\phi^{\prime} \phi^{\prime \prime}}{\mathcal{H}} \\
& +2 \kappa \phi^{\prime 2}, \\
\mathcal{M}_{\mathcal{F F}}= & \frac{\left(a^{3 / 2}\right)^{\prime \prime}}{a^{3 / 2}}+a^{2} \frac{\partial^{2} W}{\partial \chi^{2}}-\frac{2 \kappa}{3 \mathcal{H}^{2}} \mathcal{H}^{\prime} \chi^{\prime 2}+\frac{4}{3} \kappa \frac{\chi^{\prime} \chi^{\prime \prime}}{\mathcal{H}} \\
& +2 \kappa \chi^{\prime 2},
\end{aligned}
$$

$$
\begin{aligned}
\mathcal{M}_{\mathcal{F G}}= & \mathcal{M}_{G \mathcal{F}} \\
= & \frac{\partial^{2} W}{\partial \phi \partial \chi} a^{2}-\frac{2 \kappa \mathcal{H}^{\prime}}{3 \mathcal{H}^{2}} \phi^{\prime} \chi^{\prime}+\frac{2 \kappa}{3 \mathcal{H}}\left(\phi^{\prime} \chi^{\prime \prime}+\chi^{\prime} \phi^{\prime \prime}\right) \\
& +2 \kappa \phi^{\prime} \chi^{\prime} .
\end{aligned}
$$

In the limit $W(\phi, \chi) \rightarrow W(\phi)$ and $\chi^{\prime} \rightarrow 0, \mathcal{M}_{G \mathcal{F}} \rightarrow 0$ and $\mathcal{M}_{G G} \rightarrow z_{\phi}^{\prime \prime} / z_{\phi}$. Thus, the result of the single-defect system is recovered.

Defining

$$
\mathcal{L}=\left(\begin{array}{c}
\mathcal{G} \\
\mathcal{F}
\end{array}\right), \quad \mathcal{M}=\left(\begin{array}{ll}
\mathcal{M}_{G G} & \mathcal{M}_{G \mathcal{F}} \\
\mathcal{M}_{G \mathcal{F}} & \mathcal{M}_{\mathcal{F}, \mathcal{F}}
\end{array}\right)
$$

Eqs. (5.42) and (5.43) can be simply written in matrix notation:

$$
\mathcal{L}^{\prime \prime}-\square \mathcal{L}-\mathcal{M} \mathcal{L}=0
$$

It is worth noticing that the derivation presented here is fully gauge invariant. Since $\mathcal{G}$ and $\mathcal{F}$ are gauge invariant their evolution equation is the same in any specific gauge. It is therefore useful to cross-check the gauge-invariant derivation with a gauge-dependent discussion. A particularly useful coordinate system, already exploited in [20], is the off-diagonal gauge where $E=0$ and $\psi=0$. The gauge-invariant variables become, in this gauge,

$$
\begin{gathered}
\Psi=\mathcal{H} C, \quad \Xi=\xi-\mathcal{H} C-C^{\prime}, \\
\Phi=\delta \phi+\phi^{\prime} C, \quad X=\delta \chi+\chi^{\prime} C .
\end{gathered}
$$

Inserting Eqs. (5.49) and (5.50) into Eqs. (5.39) and (5.40) it is also clear that, in the off-diagonal gauge, $G=a^{3 / 2} \delta \phi$ and $\mathcal{F}=a^{3 / 2} \delta \chi$. Therefore, using the evolution equations written explicitly in the off-diagonal gauge, it is possible to recover exactly Eqs. (5.42) and (5.43). The essential steps of this exercise are summarized in the appendix.

Let us now consider, as a useful application, the determination of the localization properties of the scalar zero modes in the case of the solutions discussed in Sec. III and parametrized according to Eq. (3.1). In the case of the kinkantikink system the various matrix elements appearing in Eq. (5.47) can be computed in terms of $g$. The result of this algebraic calculation is

$$
\begin{aligned}
& \mathcal{M}_{G G}=\frac{b^{2}}{4 g^{2}}\left[g^{2}\left(1-g^{2}\right)\left(76 g^{2}+42 g+1\right)+4(11+g)^{2}(1-g)^{3}\left(6 g^{2}+g+1\right)\right], \\
& \mathcal{M}_{\mathcal{F G}}=\mathcal{M}_{G \mathcal{F}}=\frac{b^{2}}{g^{2}}\left[7 g^{2}\left(1-g^{2}\right)^{3 / 2}+\left(1-g^{2}\right)^{5 / 2}(g+1)(2 g-1)+3 g\left(1-g^{2}\right)^{2}\right], \\
& \mathcal{M}_{\mathcal{F F}}=\frac{b^{2}}{4 g^{2}}\left[g^{2}\left(1-g^{2}\right)\left(76 g^{2}-42 g+1\right)+4(1-g)^{2}(1+g)^{3}\left(6 g^{2}-g+1\right)\right],
\end{aligned}
$$


Using the explicit expression of $g(x)$ derived in Eq. (3.6) the matrix elements of $\mathcal{M}$ can be expressed as a function of the rescaled bulk coordinate $x=b w$ :

$$
\begin{aligned}
\mathcal{M}_{G \mathcal{G}} & =\frac{b^{2}}{4 x^{2}\left(x^{2}+1\right)^{5 / 2}}\left[x^{2} \sqrt{x^{2}+1}\left(77 x^{2}+42 x \sqrt{x^{2}+1}+1\right)+4\left(\sqrt{x^{2}+1}-x\right)\left(7 x^{2}+x \sqrt{x^{2}+1}+1\right)\right] \\
\mathcal{M}_{\mathcal{F G}} & =\mathcal{M}_{\mathcal{G F}}=\frac{b^{2}}{x^{2}\left(x^{2}+1\right)^{5 / 2}}\left[x\left(x^{2}+1\right)(7 x+3)+\left(x^{2}+x \sqrt{x^{2}+1}-1\right)\right] \\
\mathcal{M}_{\mathcal{F F}} & =\frac{b^{2}}{4 x^{2}\left(x^{2}+1\right)^{5 / 2}}\left[x^{2} \sqrt{x^{2}+1}\left(77 x^{2}-42 x \sqrt{x^{2}+1}+1\right)+4\left(\sqrt{x^{2}+1}+x\right)\left(7 x^{2}-x \sqrt{x^{2}+1}+1\right)\right] .
\end{aligned}
$$

To determine if the lowest mass eigenstates are normalizable or not it suffices to go see if Eqs. (5.42) and (5.43) admit normalizable solutions in the case $\square \mathcal{G}=\square \mathcal{F}=0$ when the coefficients $\mathcal{M}_{\mathcal{G G}}, \mathcal{M}_{\mathcal{F G}}$, and $\mathcal{M}_{\mathcal{F F}}$ are the ones determined in Eq. (5.52).

The idea is therefore the following. Let us solve, asymptotically, the explicit form of Eqs. (5.42) and (5.43) in the limit $x \rightarrow-\infty$. By imposing initial conditions for $x \rightarrow$ $-\infty$ the system can be integrated numerically across $x=$ 0 . This procedure will give, as a function of different initial conditions, the lowest mass eigenstates provided the obtained solution is regular and sufficiently convergent for large absolute values of the rescaled bulk coordinate.

In the limit $x \rightarrow-\infty$ it can be verified that Eq. (5.52) gives

$$
\begin{gathered}
\mathcal{M}_{G G} \simeq \frac{119}{4} \frac{b^{2}}{x^{2}}, \quad \mathcal{M}_{\mathcal{F G}}=\mathcal{M}_{G \mathcal{F}} \simeq \frac{7 b^{2}}{x^{3}}, \\
\mathcal{M}_{\mathcal{F F}}=\frac{35}{4} \frac{b^{2}}{x^{2}} .
\end{gathered}
$$

Therefore, for $x \rightarrow-\infty$, the solutions for $\mathcal{G}$ and $\mathcal{F}$ are simple power law, i.e.

$$
\begin{gathered}
\mathcal{G}_{0}(x) \simeq|x|^{\gamma_{ \pm}}, \quad \gamma_{ \pm}=\frac{1 \pm 2 \sqrt{30}}{2}, \\
\mathcal{F}_{0}(x) \simeq|x|^{\delta_{ \pm}}, \quad \delta_{+}=\frac{7}{2}, \quad \delta_{-}=-\frac{5}{2} .
\end{gathered}
$$

Recall now that the zero mode is normalizable provided

$$
\int_{-\infty}^{+\infty}\left|\mathcal{G}_{0}(x)\right|^{2} d x, \quad \int_{-\infty}^{+\infty}\left|\mathcal{F}_{0}(x)\right|^{2} d x
$$

are both convergent. This requirement implies that the only asymptotic initial conditions (for $x \rightarrow-\infty$ ) compatible with the finiteness of the integrals appearing in Eq. (5.56) are the ones parametrized in terms of $\gamma_{-}$and $\delta_{-}$. The numerical integration of the system can be performed by starting with a sufficiently negative $x_{\mathrm{i}}$, for instance $x_{\mathrm{i}}=$ $-10^{3}$. Thus, the initial conditions will be dictated by $\mathcal{G}_{0}\left(x_{\mathrm{i}}\right) \simeq\left|x_{\mathrm{i}}\right|^{\gamma_{-}}$and by $\mathcal{F}_{0}\left(x_{\mathrm{i}}\right) \simeq\left|x_{\mathrm{i}}\right|^{\delta_{-}}$. It turns out, after explicit numerical integration, that a singularity is always developed in the origin so that the obtained solution is not normalizable because of the behavior near $x=0$ where the matrix elements of $\mathcal{M}$ diverge. We then conclude that the kink-antikink system does not admit a normalizable zero mode. A similar discussion can be performed in the case of the solutions reported in Sec. IV. Also in that case the scalar zero modes are not normalizable because of the behavior of the solution in the origin.

\section{CONCLUDING REMARKS}

The possibility of gravitating multidefects from higherdimensional warped geometries has been scrutinized. It has been shown that, indeed, it is possible to find systems where qualitatively different profiles arise simultaneously. After devising a general method for the construction of multidefects solutions, specific examples, compatible with $\mathrm{AdS}_{5}$, have been presented. While the obtained solutions were only illustrative, it can be argued, on general grounds, that the following configurations can explicitly constructed:

(i) kink-antikink systems both in the case of EinsteinHilbert gravity and in the case of Gauss-Bonnet gravity;

(ii) configurations containing one topological soliton and a nontopological profile;

(iii) systems containing two baglike profiles, both nontopological.

The second class of solutions listed above corresponds, indeed, to the so-called trapping bag solutions that may be used to model (static) confining configurations in $(1+1)$ dimensions. As in the $(1+1)$-dimensional case threedefects solutions may be constructed, it is justified to speculate that the present considerations may also be extended to the case of three (or even more) defects.

As far as the localization properties of the zero modes are concerned, it has been shown that while the tensor and vector modes exhibit exactly the same features arising in the case of single gravitating defects, the scalar modes present qualitatively new features. In the case of gravitating multidefects, the fluctuations of the geometry are coupled with the fluctuations of all the profiles of the system. A fully gauge-invariant formalism for the analysis of the localization properties of the fluctuations of various spin has then been developed by extending the treatment already exploited in the case of single gravitating defects.

\section{APPENDIX: OFF-DIAGONAL GAUGE}

In this appendix the decoupled set of evolution equations for the scalar modes of the geometry will be derived in the 
off-diagonal gauge. The reported exercise complements and corroborates the gauge-invariant derivation reported in Sec. V. The rationale for the previous statement is that since the canonical (scalar) normal modes defined in Eqs. (5.39) and (5.40) are gauge invariant, they must also obey the same evolution equations [i.e. Eqs. (5.42) and (5.43)] in any specific gauge. As observed in Sec. V, in the off-diagonal gauge, i.e. the gauge where $\psi=E=0, G=$ $a^{3 / 2} \delta \phi$ and $\mathcal{F}=a^{3 / 2} \delta \chi$. The exercise presented here will then be to derive Eqs. (5.42) and (5.43) by using directly the evolution equations written in the off-diagonal gauge.

The evolution equations in the off-diagonal gauge can be obtained by expressing the gauge-invariant quantities of Eqs. (5.26), (5.27), (5.28), and (5.29) in the gauge $\psi=0$ and $E=0$. The result of this procedure is given by Eqs. (5.49) and (5.50). Using Eqs. (5.49) and (5.50) into Eqs. (5.30) and (5.31) we get

$$
\begin{gathered}
\mathcal{H} \xi^{\prime}+2\left(\mathcal{H}^{\prime}+3 \mathcal{H}^{2}\right) \xi-\mathcal{H} \square C \\
+\frac{2}{3} \kappa a^{2}\left[\frac{\partial W}{\partial \phi} \delta \phi+\frac{\partial W}{\partial \chi} \delta \chi\right]=0, \\
\mathcal{H} \square C+\square C^{\prime}-4 \mathcal{H} \xi^{\prime}-\square \xi-2 \kappa\left[\phi \delta \phi^{\prime}+\chi^{\prime} \delta \chi^{\prime}\right] \\
-\frac{4}{3} \kappa a^{2} W \xi+\frac{2}{3} \kappa a^{2}\left(\frac{\partial W}{\partial \phi} \delta \phi+\frac{\partial W}{\partial \chi} \delta \chi\right) .
\end{gathered}
$$

Using Eqs. (5.49) and (5.50) into Eqs. (5.32) and (5.33) the following pair of conditions are obtained:

$$
\begin{gathered}
\xi-C^{\prime}-3 \mathcal{H} C=0, \\
3 \mathcal{H} \xi=-\kappa\left(\phi^{\prime} \delta \phi+\chi^{\prime} \delta \chi\right) .
\end{gathered}
$$

Finally, using Eqs. (5.49) and (5.50) into Eqs. (5.28) and (5.29) we do get

$$
\begin{aligned}
\delta \phi^{\prime \prime}+ & 3 \mathcal{H} \delta \phi^{\prime}-\square \delta \phi-\frac{\partial^{2} W}{\partial \phi^{2}} a^{2} \delta \phi-\frac{\partial^{2} W}{\partial \phi \partial \chi} a^{2} \delta \chi \\
+ & \left(\xi^{\prime}-\square C\right) \phi^{\prime}+2\left(\phi^{\prime \prime}+3 \mathcal{H} \phi^{\prime}\right) \xi=0, \\
\delta \chi^{\prime \prime}+ & 3 \mathcal{H} \delta \chi^{\prime}-\square \delta \chi-\frac{\partial^{2} W}{\partial \chi^{2}} a^{2} \delta \chi-\frac{\partial^{2} W}{\partial \phi \partial \chi} a^{2} \delta \phi \\
+ & \left(\xi^{\prime}-\square C\right) \chi^{\prime}+2\left(\chi^{\prime \prime}+3 \mathcal{H} \chi^{\prime}\right) \xi=0 .
\end{aligned}
$$

As already mentioned, in the off-diagonal gauge the variables $\mathcal{G}$ and $\mathcal{F}$ become

$$
\mathcal{G}=a^{3 / 2} \delta \phi, \quad \mathcal{F}=a^{3 / 2} \delta \chi .
$$

Subtracting Eqs. (A2) from Eq. (A1) and recalling Eq. (5.33) we get

$$
\begin{gathered}
\square C-\xi^{\prime}=-\frac{2 \kappa}{3 \mathcal{H}}\left[\phi^{\prime} \delta \phi^{\prime}+\chi^{\prime} \delta \chi^{\prime}\right]-2 \xi^{\prime}, \\
\xi=-\frac{\kappa}{3 \mathcal{H}}\left[\phi^{\prime} \delta \phi+\chi^{\prime} \delta \chi\right] .
\end{gathered}
$$

By inserting Eq. (A7) into Eqs. (A5) and (A6), $\delta \phi$ and $\delta \chi$ can be completely eliminated in favor of $\mathcal{G}$ and $\mathcal{F}$. In Eqs. (A5) and (A6) the only remaining pieces will be, respectively, the expressions proportional to $\left(\square C-\xi^{\prime}\right)$ and to $\xi$ :

$$
\begin{gathered}
\frac{1}{a^{3 / 2}}\left\{\mathcal{G}^{\prime \prime}-\square \mathcal{G}-\left[\frac{\left(a^{3 / 2}\right)^{\prime \prime}}{a^{3 / 2}}+\frac{\partial^{2} W}{\partial \phi^{2}} a^{2}\right]-\frac{\partial^{2} W}{\partial \phi \partial \chi} \mathcal{F}\right\} \\
+\left(\xi^{\prime}-\square C\right) \phi^{\prime}+2\left(\phi^{\prime \prime}+3 \mathcal{H} \phi^{\prime}\right) \xi=0, \\
\frac{1}{a^{3 / 2}}\left\{\mathcal{F}^{\prime \prime}-\square \mathcal{F}-\left[\frac{\left(a^{3 / 2}\right)^{\prime \prime}}{a^{3 / 2}}+\frac{\partial^{2} W}{\partial \chi^{2}} a^{2}\right]-\frac{\partial^{2} W}{\partial \chi \partial \phi} G\right\} \\
+\left(\xi^{\prime}-\square C\right) \chi^{\prime}+2\left(\chi^{\prime \prime}+3 \mathcal{H} \chi^{\prime}\right) \xi=0 .
\end{gathered}
$$

But using Eq. (A7) into Eqs. (A8) and (A9) we get

$$
\square C-\xi^{\prime}=\frac{2 \kappa}{3 \mathcal{H} a^{3 / 2}}\left[\phi^{\prime \prime} \mathcal{G}+\chi^{\prime \prime} \mathcal{F}-\frac{\mathcal{H}^{\prime}}{\mathcal{H}}\left(\phi^{\prime} \mathcal{G}+\chi^{\prime} \mathcal{F}\right)\right]
$$

$$
\xi=-\frac{\kappa}{3 \mathcal{H} a^{3 / 2}}\left(\phi^{\prime} \mathcal{G}+\chi^{\prime} \mathcal{F}\right) .
$$

Equations (A12) and (A13) show that $\left(\square C-\xi^{\prime}\right)$ and to $\xi$ can be solely expressed in terms of $\mathcal{G}, \mathcal{F}$ and their first derivatives with respect to the bulk coordinate. This completes the derivation since inserting then Eqs. (A12) and (A13) into Eqs. (A10) and (A11), Eqs. (5.42) and (5.43) are readily obtained.
[1] R. Rajaraman, Solitons and Instantons (Elsevier, Amsterdam, 1982).

[2] R. Rajaraman and E. Weinberg, Phys. Rev. D 11, 2950 (1975).

[3] C. Montonen, Nucl. Phys. B112, 349 (1976).

[4] S. Coleman, Aspects of Symmetry: Selected Erice Lectures (Cambridge University press, Cambridge, England, 1985).
[5] J. Rubinstein, J. Math. Phys. (N.Y.) 11, 258 (1970).

[6] J. R. Morris, Phys. Rev. D 51, 697 (1995).

[7] D. Bazeia, R. F. Ribeiro, and M. M. Santos, Phys. Rev. D 54, 1852 (1996).

[8] D. Bazeia and F. A. Brito, Phys. Rev. D 61, 105019 (2000).

[9] D. Bazeia, L. Losano, and C. Wotzasek, Phys. Rev. D 66, 105025 (2002). 
[10] A. de Souza Doutra, Phys. Lett. B 626, 249 (2005).

[11] T.D. Lee, Particle Physics and Introduction to Field Theory (Harwood Academic Publisher, Chur, Switzerland, 1986), Vol. 1, p. 117.

[12] A. Chodos, R. L. Jaffe, K. Johnson, C. B. Thorn, and V. F. Weisskopf, Phys. Rev. D 9, 3471 (1974).

[13] V. A. Rubakov and M. E. Shaposhnikov, Phys. Lett. 125B, 136 (1983).

[14] V. A. Rubakov, Usp. Fiz. Nauk 171, 913 (2001) [Phys. Usp. 44, 871 (2001)].

[15] A. Kehagias and K. Tamvakis, Phys. Lett. B 504, 38 (2001).

[16] A. Kehagias and K. Tamvakis, Mod. Phys. Lett. A 17, 1767 (2002).

[17] M. Gremm, Phys. Lett. B 478, 434 (2000).

[18] M. Gremm, Phys. Rev. D 62, 044017 (2000).

[19] M. Giovannini, Phys. Rev. D 64, 064023 (2001).

[20] M. Giovannini, Phys. Rev. D 65, 064008 (2002).

[21] R. Koley and S. Kar, Classical Quantum Gravity 22, 753 (2005).

[22] S. Pal and S. Kar, Classical Quantum Gravity 23, 2571 (2006).

[23] C. Bogdanos, A. Dimitriadis, and K. Tamvakis, Phys. Rev. D 74, 045003 (2006).

[24] K. Farakos and P. Pasipoularides, Phys. Lett. B 621, 224 (2005).

[25] K. Farakos and P. Pasipoularides, hep-th/0609089.

[26] O. Corradini and Z. Kakushadze, Phys. Lett. B 494, 302 (2000).

[27] N. E. Mavromatos and J. Rizos, Phys. Rev. D 62, 124004 (2000).

[28] M. Giovannini, Phys. Rev. D 63, 064011 (2001).

[29] M. Giovannini, Phys. Rev. D 64, 124004 (2001).

[30] N. Deruelle and C. Germani, Nuovo Cimento Soc. Ital. Fis. B 118, 977 (2003).

[31] O. Corradini and Z. Kakushadze, Phys. Lett. B 506, 167 (2001).

[32] O. Corradini, A. Iglesias, Z. Kakushadze, and P. Lang- felder, Phys. Lett. B 521, 96 (2001).

[33] M. Giovannini, H. Meyer, and M. E. Shaposhnikov, Nucl. Phys. B619, 615 (2001).

[34] M. Giovannini, Classical Quantum Gravity 23, L73 (2006).

[35] L. Randall and R. Sundrum, Phys. Rev. Lett. 83, 3370 (1999).

[36] L. Randall and R. Sundrum, Phys. Rev. Lett. 83, 4690 (1999).

[37] M. Giovannini, Classical Quantum Gravity 20, 1063 (2003).

[38] S. Randjbar-Daemi and M. Shaposhnikov, Nucl. Phys. B645, 188 (2002).

[39] S. Randjbar-Daemi, Mod. Phys. Lett. A 18, 2459 (2003).

[40] M. Shaposhnikov, P. Tinyakov, and K. Zuleta, J. High Energy Phys. 09 (2005) 062.

[41] M. Giovannini, Phys. Rev. D 74, 087505 (2006).

[42] C. Lanczos, Z. Phys. 73, 147 (1932); Ann. Math. 39, 842 (1938).

[43] D. Lovelock, J. Math. Phys. (N.Y.) 12, 498 (1971).

[44] J. Madore, Phys. Lett. 110A, 289 (1985).

[45] J. Madore, Phys. Lett. 111A, 283 (1985).

[46] B. Zwiebach, Phys. Lett. 156B, 315 (1985).

[47] D. G. Boulware and S. Deser, Phys. Rev. Lett. 55, 2656 (1985); Phys. Lett. B 175, 409 (1986).

[48] R. R. Metsaev and A. A. Tseytlin, Phys. Lett. B 191, 354 (1987); Nucl. Phys. B293, 385 (1987).

[49] C. G. Callan, E. J. Martinec, M. J. Perry, and D. Friedan, Nucl. Phys. B262, 593 (1985).

[50] A. Sen, Phys. Rev. Lett. 55, 1846 (1985).

[51] N. Deruelle and J. Madore, gr-qc/0305004.

[52] M. Giovannini, Int. J. Mod. Phys. D 11, 1209 (2002).

[53] M. Giovannini, Phys. Rev. D 66, 044016 (2002).

[54] M. Giovannini, J. V. Le Be, and S. Riederer, Classical Quantum Gravity 19, 3357 (2002).

[55] J. M. Cline, J. Descheneau, M. Giovannini, and J. Vinet, J. High Energy Phys. 06 (2003) 048. 